\title{
UNIVERSITYOF
}

FORWARD

THINKING

WESTMINSTER用

WestminsterResearch

http://www.westminster.ac.uk/westminsterresearch

\section{A Fully Planar Substrate Integrated Probe-Based Wideband Orthomode Transducer}

Jankovic, U. and Budimir, D.

This is a copy of the author's accepted version of a paper subsequently to be published in the proceedings of the 12th European Conference on Antennas and Propagation (EUCAP), Excel, London, 09 to 13 Apr 2018, IEEE.

The final published version will be available online at:

\section{https://doi.org/10.1049/cp.2018.0427}

(C) 2018 IEEE . Personal use of this material is permitted. Permission from IEEE must be obtained for all other uses, in any current or future media, including reprinting/republishing this material for advertising or promotional purposes, creating new collective works, for resale or redistribution to servers or lists, or reuse of any copyrighted component of this work in other works.

The WestminsterResearch online digital archive at the University of Westminster aims to make the research output of the University available to a wider audience. Copyright and Moral Rights remain with the authors and/or copyright owners.

Whilst further distribution of specific materials from within this archive is forbidden, you may freely distribute the URL of WestminsterResearch: ((http://westminsterresearch.wmin.ac.uk/)).

In case of abuse or copyright appearing without permission e-mail repository@westminster.ac.uk 


\title{
A Fully Planar Substrate Integrated Probe-Based Wideband Orthomode Transducer
}

\author{
Uros Jankovic $^{1}$, Djuradj Budimir ${ }^{1,2}$ \\ ${ }^{1}$ Wireless Communications Research Group, University of Westminster, London, UK, \\ uros.jankovic@my.westminster.ac.uk,d.budimir@westminster.ac.uk \\ ${ }^{2}$ School of Electrical Engineering, University of Belgrade, Belgrade, Serbia
}

\begin{abstract}
This paper introduces a planar orthomode transducer (OMT) using substrate integrated waveguides (SIWs) and substrate integrated coaxial lines (SICLs) that is entirely contained within two substrate layers. SICLs form a quadruplet of probes inside the metal waveguide with fully symmetrical geometry. Such a structure is smaller and lighter than a waveguide OMT, its construction is simpler than of a coaxial OMT, however, isolation and loss properties are less degraded compared to the use of planar unshielded technologies such as microstrip. A new wideband inline SICL to SIW transition has been developed to allow grade separation of SIWs belonging to opposite polarizations through different substrate layers. The designed substrate integrated probe-based OMT on $\mathrm{RO4003C}^{\mathrm{TM}}$ substrate covers the frequency band from 8.1 to $12.5 \mathrm{GHz}$ with return loss higher than $19 \mathrm{~dB}$, insertion loss less than $1.2 \mathrm{~dB}$, inter-port isolation higher than $62 \mathrm{~dB}$ and cross-polarization discrimination higher than $65 \mathrm{~dB}$.
\end{abstract}

Index Terms-orthomode transducer (OMT), planar, substrate integrated coaxial line (SICL), substrate integrated waveguide (SIW), transition.

\section{INTRODUCTION}

Orthomode transducers (OMTs) are microwave components used for separating/exciting two signals transferred inside metal waveguides within a pair or orthogonally polarized dominant degenerate modes. They are widely found in applications like wireless communications and radiometry, especially in radio astronomy receivers. Waveguide OMT implementations use 4-way junctions [1], [2] (2 output branches), 5-way Bøifot junctions [3], [4] (3 output branches) and 6-way turnstile junctions [5] - [7] (4 output branches). However, although bringing quality in terms of low losses and power handling capacity, these allwaveguide components are naturally large, heavy and expensive solutions, unless they are used at very high frequencies. This problem gets worse as the wideband isolation and polarization purity are improved by adding more side branches coming out of the main dual-polarized waveguide, thus increasing the level of symmetry.

Coaxial (probe) transducers like [8] are less bulky, but still relatively complex and expensive solutions. They can also spread over a longer section of the main waveguide such in [9], even though this additional space can be utilized to increase the bandwidth using finlines or ridges [10]. OMTs in [11] and [12] are planar inside the waveguide, but still have vast external circuitry for combining signals. Moreover, [12] has several orthogonal PCB elements inside the main waveguide. Fully planar OMTs [13], [14] and [15] are relatively narrowband. Although of a simple construction, [13] does not have full symmetry and microstrip circuits increase losses. The OMT in [15] is very compact, but its integration with the radiating elements limits flexibility, especially for higher performance applications.

The proposed OMT is entirely planar, including line transitions and power combining microwave circuits. It is implemented through shielded substrate integrated waveguides and rectangular coaxial lines, hence practically eliminating radiation losses and spurious couplings. Apart from manufacturing advantages, a PCB OMT design allows direct integration with other planar circuit components. There is a "full symmetry" inside the main waveguide - 4 planes of symmetry, having an identical probe coming out of each of 4 waveguide side walls. Thus, the inherent symmetry of the square shape of the waveguide is preserved and isolation and cross-polarization minimized. Other waveguide shapes with at least four planes of symmetry, most notably the circular one, can directly replace the square one and minimal parameter changes are required to optimize the performance. Another essential attribute of the OMT is its fractional bandwidth wider than $44 \%$, which required wideband design of each of its elements.

\section{OMT STRUCTURE}

The OMT is implemented inside a two-layered PCB inserted transversally into metal square waveguide having common physical port with two orthogonal polarizations at one end and short termination at the other. As the PCB substrate is chosen $0.81 \mathrm{~mm}$ thick ceramic based Rogers Corporation RO4003C for its favourable fabrication properties at the cost of higher losses than with laminates based on PTFE composites. A layout of the OMT is given in Fig. 1.

The footprint of the OMT can in fact be reduced in size if the substrate integrated lines are positioned tighter with the main metal waveguide inner walls. However, these areas are left for bolts to go through and connect together the stacked structure, providing good contact between the conductive surfaces of PCB layers and waveguide sections. 
Another reason is to reduce the number of corner bends of the lines and diminish reflections.

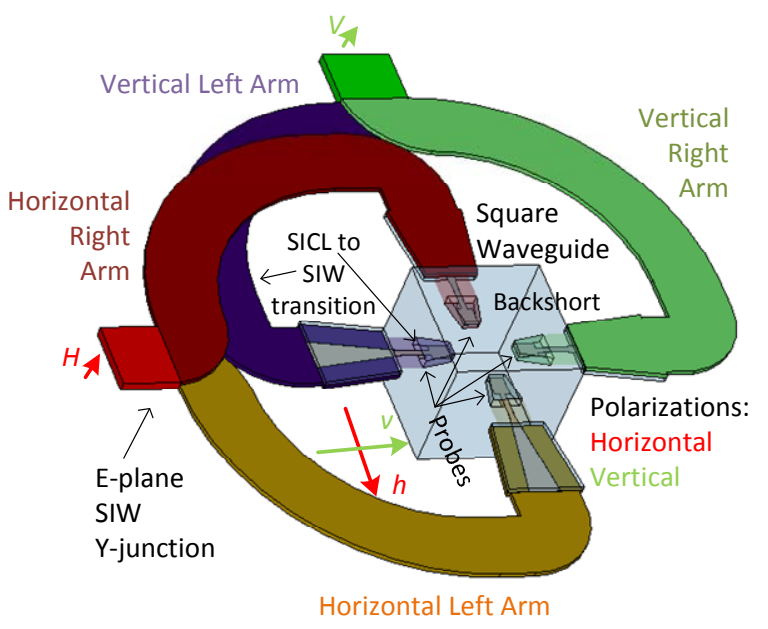

Fig. 1. Layout of the proposed substrate integrated probe-based wideband orthomode transducer.

The presented design is also scalable. For example, it can be made more compact by using three probes and shorter SICL to SIW transition tapers at the cost of worse isolation, polarization purity as well as smaller bandwidth. On the opposite side, it can be made very wideband by using ridged square waveguide.

\section{A. Probes}

The coupling between the side lines and the main waveguide supporting propagation of orthogonally polarized modes is made through balanced substrate integrated coaxial electric probes (Fig. 2). They have good wideband characteristics even with electrically thin substrate, which is a considerable problem if a turnstile waveguide junction is tried to be implemented in this way.

Since the width of a substrate integrated coaxial line (SICL) outer conductor has negligible effect on the dominant TEM mode, and directly determines the first upper waveguide mode [16], it was chosen to be $a_{\mathrm{SICL}}=5.2 \mathrm{~mm}$ so that the $\mathrm{TE}_{10}$ cut off frequency is significantly above the $\mathrm{X}$ band. With the substrate height fixed by the PCB laminate thickness, the flat inner conductor width was selected to be $W_{\mathrm{COND}}=1.0 \mathrm{~mm}$ in order to have characteristic impedance around $50 \Omega$ and relatively easy fabrication regarding tolerance. In turn, $W_{\text {COND }}$ has miniscule effect on $\mathrm{TE}_{10}$ mode (slightly lowering its cutoff frequency as it gets wider).

The probes have been optimized having wider inner conductor ends to improve overall matching and with ground plane stubs parallel to the inner conductors, having the roll of improving the matching at the higher frequency side of the passband. Optimization process could be made almost four times faster by using two symmetry planes - PMC along the center E-plane of the square waveguide and PEC along its $\mathrm{H}$-plane relative to the polarization of the selected input $\mathrm{TE}_{10}$ mode. Furthermore, the square waveguide is closed with a quarter-wave short circuit stub on top of the cross section containing probes, with its exact length also being an optimization variable.

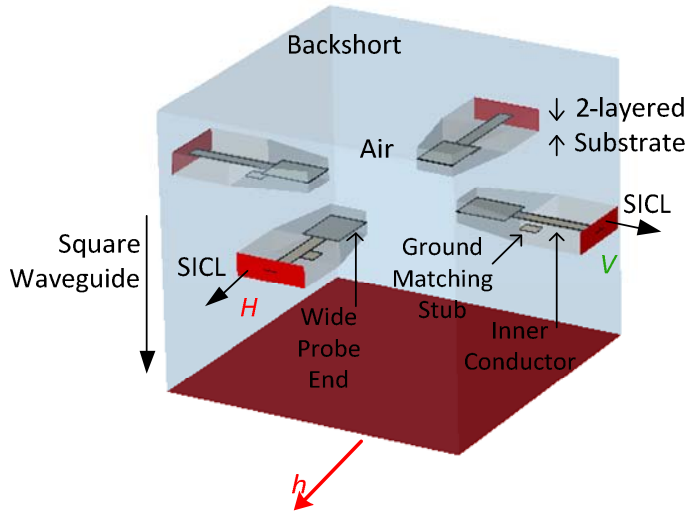

Fig. 2. Input short circuited square waveguide with SICL probes transversally placed in.

Dielectric inside the main waveguide follows the shapes of the planar metal probes, both to give mechanical support and to provide smoother transition between the SICLs and the hollow square metal waveguide interfaces. Removal of the rest of the substrate inside the waveguide reduces dielectric losses.

\section{B. SICL to SIW transitions}

Apart from realizing SICLs, the second substrate layer is needed because intersection of arms belonging to different polarizations within the same layer (Vertical Left Arm and Horizontal Right Arm in Fig. 1) has to be avoided. Substrate integrated waveguide (SIW) was selected to be used in the arms crossing over region as a shielded and low loss single layer guided wave structure. Thus, there was a need for developing a wideband inline SICL to SIW transition (Fig. 3).

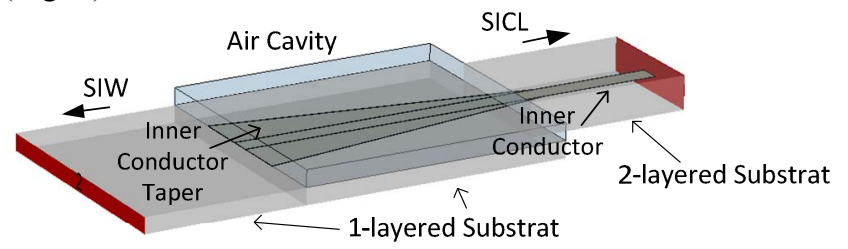

Fig. 3. Wideband double layer SICL to single layer SIW transition.

The central piece of the transition is the linearly tapered metallization between the two PCB layers, starting as the narrow SICL inner conductor and spreading to the full width of SIW broad wall. The transition medium is piece-wise homogeneous. Only the bottom layer is the laminate, whereas the upper one is air (filling the volume of the laminate that had been cut out). This way, the signal is guided through only one substrate layer, without strong reflection coming from the other layer. There is resemblance with a microstrip taper, both in the way how it is connected to SICL and SIW as well as having inhomogeneous dielectric. However, unlike microstrip taper, this SICL to SIW transition is shielded from all four sides. 


\section{Power Combiners}

The proposed design makes possible to route both arms of one polarization through the same layer or have them in different layers. This is controlled by selecting the layer in which SIW is coupled through the transition from SICL. An interesting aspect is that if same polarization (horizontal or vertical) SIWs are in unchanged layer, the signals enter arms from the transitions having opposite phases $\left(180^{\circ}\right.$ phase difference), whereas if these SIWs are in different layers, the signals are in phase $\left(0^{\circ}\right.$ phase difference $)$.

A number of different H-plane (narrow-wall) and Eplane (broad-wall) power dividers and hybrid directional couplers, in conjunction with phase shifters where needed, have been considered as candidates for power combining role in the OMT. As the unmatched best solution has been found the E-plane straight Y-junction (Fig. 4), being very compact and, even more importantly, not having any wideband limitations whatsoever in the usable frequency range as the result of the symmetries. (Each of the complete 4 arms, from the SICL probe to the power divider SIW port, can be transformed one into another through composition of two plane symmetries.) It is, essentially, substrate integrated equivalent of the power dividers used in turnstile junction OMTs [5-7]. All other tested solutions, over a frequency range, have at least certain unneglectable level of unequal amplitude and phase distribution of signals at the end of the SIW arms, which more or less noticeably affects performance of a wideband OMT. Furthermore, they either inherently cannot cover the entire OMT band based on their mechanism of operation, having sharp degradation of performance within its limits, or if the order of a directional coupler can be increased, its size becomes significant.

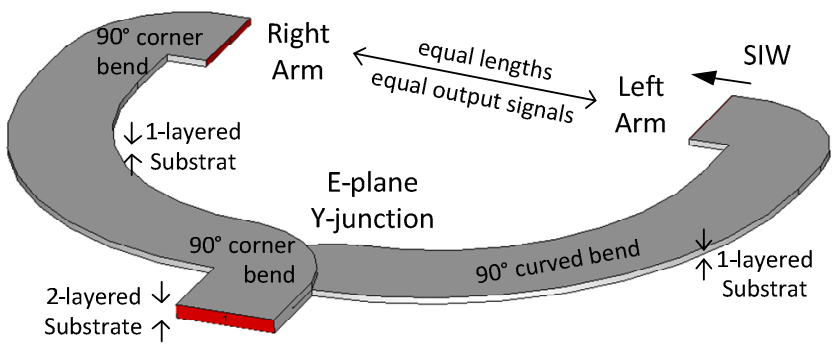

Fig. 4. Power combining circuit with SIW E-plane straight Y-junction.

\section{RESULTS}

SIWs and SICLs were designed with the following constraints regarding vias: $d<\lambda_{\mathrm{g}} / 5$ and $p<2 d$, where $d$ is the via diameter, $p$ is via pitch (distance between adjacent vias), and $\lambda_{\mathrm{g}}$ is the guided wavelength. The diameter for all the OMT vias was selected to be $d=0.6 \mathrm{~mm}$, and the via pitch to be flexible in order to have the first and the last via of a wall at particular locations. However, the number of vias in a wall was determined so as to have the distance between the consecutive vias around $0.4 \mathrm{~mm}$.

In Fig. 5 are given the layouts of the OMT PCB layers, and the numerical values of their dimensions are in Table I.
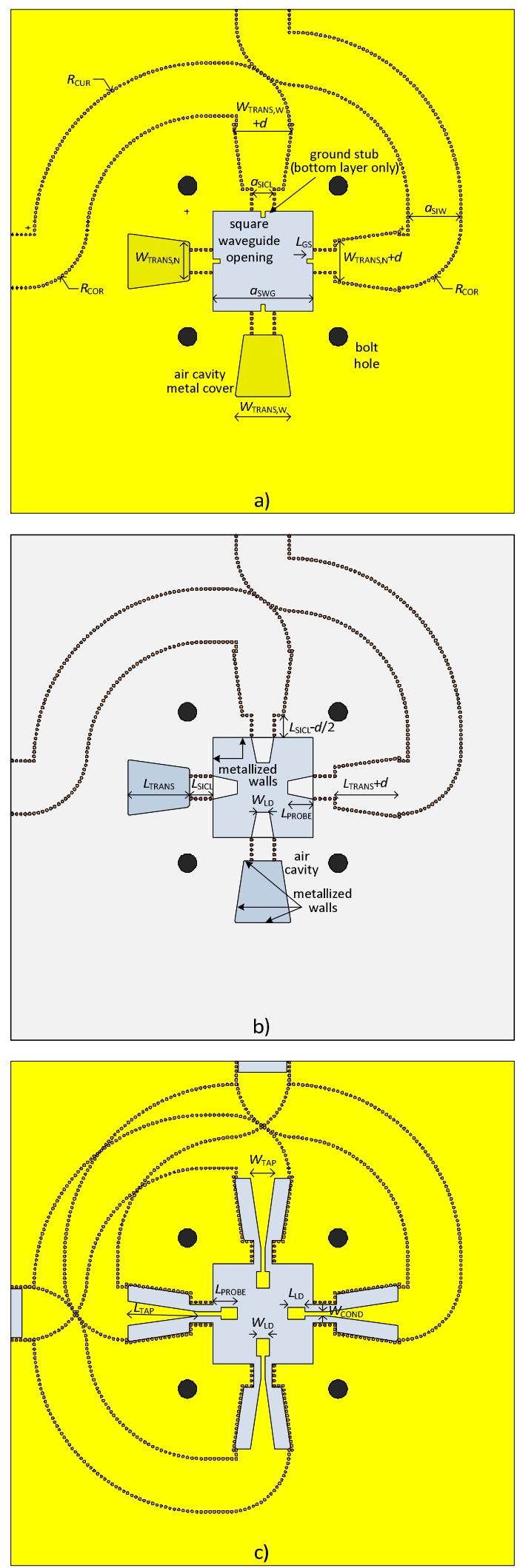

Fig. 5. PCB layouts of the wideband planar substrate integrated OMT: a) top/bottom metallization, b) top/bottom substrate and c) central metallization layer. 
In addition, the length of the square waveguide backshort is $L_{\text {SWGs }}=6.7 \mathrm{~mm}$. Except for the tuning stubs, the top and bottom substrate layer are mutually symmetrical with respect to the diagonal as the axis of symmetry. (Consequently, the central metal layer has the diagonal as its own axis of symmetry.)

\begin{tabular}{|cc|cc|}
\multicolumn{5}{c}{ Table I. OMT dimensions in mm } \\
\hline$a_{\mathrm{SWG}}$ & 22.9 & $L_{\mathrm{PROBE}}$ & 5.7 \\
$a_{\mathrm{SIW}}$ & 12.1 & $L_{\mathrm{LD}}$ & 3.9 \\
$a_{\mathrm{SICL}}$ & 5.2 & $W_{\mathrm{LD}}$ & 2.9 \\
$L_{\mathrm{SICL}}$ & 5.3 & $W_{\mathrm{COND}}$ & 1.0 \\
$L_{\mathrm{TRANS}}$ & 14.1 & $L_{\mathrm{TAP}}$ & 16.0 \\
$W_{\text {TRANS, N }}$ & 8.7 & $W_{\mathrm{TAP}}$ & 5.5 \\
$W_{\text {TRANS, }}$ & 12.8 & $R_{\mathrm{COR}}$ & 14.1 \\
$L_{\mathrm{GS}}$ & 1.5 & $R_{\mathrm{CUR}}$ & 33.8 \\
\hline
\end{tabular}

Since the structures with long via fences are computationally intensive, this OMT has practical advantage coming from its shielded nature that its operation can be simulated relatively accurately piece by piece, and the total response can be found as the response of the microwave circuit having the aforementioned parts as is components.

Fig. 6 gives the S-parameters calculated in CST Microwave Studio of the OMT accurately modelled with line walls made out vias, lossy RO4003C dielectric and lossy copper metallization that takes the surface roughness into account. The insertion loss does not exceed $1.2 \mathrm{~dB}$ in the frequency band between 8.07 and $12.53 \mathrm{GHz}$. In the same band, return loss is better than $19 \mathrm{~dB}$. If narrow spikes attributed to numerical errors are disregarded, crosspolarization discrimination is higher than $65 \mathrm{~dB}$ and interport isolation higher than $62 \mathrm{~dB}$ within this span.
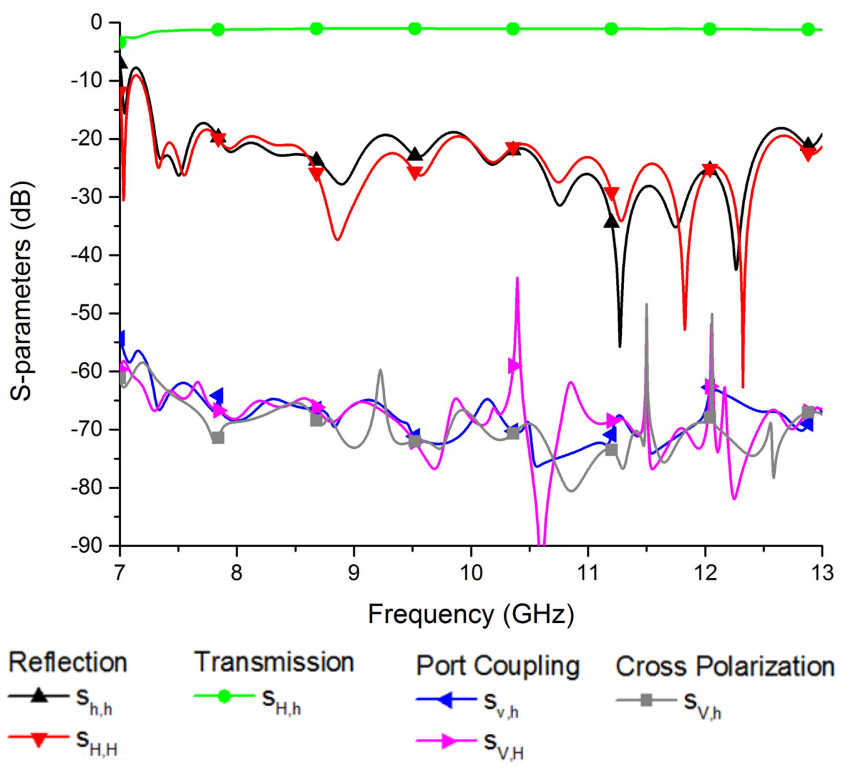

Fig. 6. Return loss (RL), insertion loss (IL), inter-port isolation (IPI) and cross-polarization discrimination (XPD) characteristics of the proposed OMT.

\section{CONCLUSION}

A novel, wholly planar OMT in mixed SIW/SICL technology has been presented. The square waveguide carrying two orthogonal polarizations is fed by four SICL probes of the OMT. Tapered center conductor sections in nonhomogeneous dielectrics are used for inline transitions between SICLs and single layer SIWs. Finally, each pair of signals belonging to the same polarization and travelling through two branches of SIW is combined in an E-plane straight Y-junction. Excellent wideband, high isolation and polarization purity performances have been achieved wider than in standard WR $90 \mathrm{X}$ band rectangular waveguide recommended frequency range.

\section{ACKNOWLEDGMENT}

This work was supported by UKIERI University Grants Commission (grant IND/CONT/G/16-17/63).

\section{REFERENCES}

[1] J. M. Rebollar, J. Esteban, and J. D. Frutos, "A dual frequency OMT in the $\mathrm{Ku}$ band for TT amp;C applications," in IEEE Antennas and Propagation Society International Symposium. 1998 Digest. Antennas: Gateways to the Global Network. Held in conjunction with: USNC/URSI National Radio Science Meeting (Cat. No.98CH36, 1998, vol. 4, pp. 2258-2261 vol.4.

[2] T. J. Reck and G. Chattopadhyay, "A $600 \mathrm{GHz}$ Asymmetrical Orthogonal Mode Transducer," IEEE Microw. Wirel. Compon. Lett., vol. 23, no. 11, pp. 569-571, Nov. 2013.

[3] A. M. Boifot, E. Lier, and T. Schaug-Pettersen, "Simple and broadband orthomode transducer (antenna feed)," Antennas Propag. IEE Proc. H - Microw., vol. 137, no. 6, pp. 396-400, Dec. 1990.

[4] C. A. Leal-Sevillano, T. J. Reck, G. Chattopadhyay, J. A. Ruiz-Cruz, J. R. Montejo-Garai, and J. M. Rebollar, "Development of a Wideband Compact Orthomode Transducer for the $180-270 \mathrm{GHz}$ Band," IEEE Trans. Terahertz Sci. Technol., vol. 4, no. 5, pp. 634636, Sep. 2014

[5] Y. Aramaki, N. Yoneda, M. Miyazaki, and T. Horie, "Ultra-thin broadband OMT with turnstile junction," IEEE MTT-S International Microwave Symposium Digest, 2003, 2003, vol. 1, pp. 47-50 vol.1.

[6] A. Navarrini and R. L. Plambeck, "A turnstile junction waveguide orthomode transducer," IEEE Trans. Microw. Theory Tech., vol. 54, no. 1, pp. 272-277, Jan. 2006.

[7] G. Pisano et al., "A Broadband WR10 Turnstile Junction Orthomode Transducer," IEEE Microw. Wirel. Compon. Lett., vol. 17, no. 4, pp. 286-288, Apr. 2007.

[8] G. Engargiola and A. Navarrini, "K-band orthomode transducer with waveguide ports and balanced coaxial probes," IEEE Trans. Microw. Theory Tech., vol. 53, no. 5, pp. 1792-1801, May 2005.

[9] M. A. Moharram, A. Mahmoud, and A. A. Kishk, "A Simple Coaxial to Circular Waveguide OMT for Low-Power Dual-Polarized Antenna Applications," IEEE Trans. Microw. Theory Tech., vol. PP, no. 99, pp. 1-7, 2017.

[10] S. J. Skinner and G. L. James, "Wide-band orthomode transducers," IEEE Trans. Microw. Theory Tech., vol. 39, no. 2, pp. 294-300, Feb. 1991.

[11] P. K. Grimes, O. G. King, G. Yassin, and M. E. Jones, "Compact broadband planar orthomode transducer," Electron. Lett., vol. 43, no. 21, pp. 1146-1147, Oct. 2007.

[12] Y. Tao and Z. Shen, "Design of broadband planar orthomode transducers using substrate integrated waveguide," 2008 Asia-Pacific Microwave Conference, 2008, pp. 1-4. 
[13] R. W. Jackson, "A planar orthomode transducer," IEEE Microw. Wirel. Compon. Lett., vol. 11, no. 12, pp. 483-485, Dec. 2001.

[14] M. K. Mandal, K. Wu, and D. Deslandes, "A compact planar orthomode transducer," 2011 IEEE MTT-S International Microwave Symposium, 2011, pp. 1-4.

[15] M. Esquius-Morote, M. Mattes, and J. R. Mosig, "Orthomode Transducer and Dual-Polarized Horn Antenna in Substrate Integrated Technology," IEEE Trans. Antennas Propag., vol. 62, no. 10, pp. 4935-4944, Oct. 2014.

[16] F. Gatti, M. Bozzi, L. Perregrini, K. Wu, and R. G. Bosisio, "A Novel Substrate Integrated Coaxial Line (SICL) for Wide-Band Applications," in 2006 European Microwave Conference, 2006, pp. 1614-1617. 